\title{
Compact objects from gravitational collapse: an analytical toy model
}

\author{
Daniele Malafarina ${ }^{1, a}$, Pankaj S. Joshi ${ }^{2, b}$ \\ ${ }^{1}$ Department of Physics, Nazarbayev University, 53 Kabanbay Batyr Avenue, 010000 Astana, Kazakhstan \\ 2 Tata Institute of Fundamental Research, Homi Bhabha Road, Colaba, Mumbai 400005, India
}

Received: 4 September 2015 / Accepted: 28 November 2015 / Published online: 16 December 2015

(C) The Author(s) 2015. This article is published with open access at Springerlink.com

\begin{abstract}
We develop here a procedure to obtain regular static configurations resulting from dynamical gravitational collapse of a massive matter cloud in general relativity. Under certain general physical assumptions for the collapsing cloud, we find the class of dynamical models that lead to an equilibrium configuration. To illustrate this, we provide a class of perfect fluid collapse models that lead to a static constant density object as limit. We suggest that similar models might possibly constitute the basis for the description of formation of compact objects in nature.
\end{abstract}

\section{Introduction}

We know today that in nature compact objects such as white dwarfs and neutron stars do form from gravitational collapse of massive stars that finally settle into a regular, stationary configuration. Typically, such a compact source that results from collapse is supported by nuclear or electromagnetic forces in terms of internal pressures, but we do know that gravity plays a crucial role both in the process of collapse itself as well as in the final equilibrium configuration. This is true also for larger entities in the universe, such as galaxies and clusters of galaxies that may have a very massive compact core at the center. Therefore it is useful to investigate the role played by gravity in the formation of compact gravitating objects. The general theory of relativity constitutes the natural framework for such a study whenever the gravitational field becomes strong (i.e. when the size of the object approaches its Schwarzschild radius).

In fact, static regular perfect fluid compact sources in general relativity have been used to model very dense objects such as neutron stars for a long time now (see for example [1] or [2] and references therein). Furthermore a theory of rel-

\footnotetext{
${ }^{a}$ e-mail: daniele.malafarina@nu.edu.kz

be-mail: psj@tifr.res.in
}

ativistic elasticity provides the first step towards a physically meaningful description of the inner structure of such objects [3]. Also relativistic models for compact sources of several kinds have been proposed as description of yet to be found astrophysical exotic objects. These vary from boson stars (see [4-6] and references therein), to gravastars (see [7,8]), preon stars (see for example [9]) and quark stars (see for example [10-13]). Even though their existence is not proven the search for theoretical models that describe exotic compact objects is very important since it is related to many of the unsolved problems of modern astrophysics, such as dark matter, dark energy, the formation of structures in the universe, and the final fate of gravitational collapse of massive stars.

While the astrophysical aspects of such general relativistic models are very much under discussion and further investigation is needed, we certainly know that the physical process that gives rise to neutron stars in nature is the gravitational collapse of a massive star, somewhere in the range of 4-40 solar masses. In such a collapse process that takes place at the very end of the life-cycle of a massive star, the outer layers of the star are blasted away in a supernova explosion while the inner heavy core implodes to form a neutron star, a very high density object of the radius of the order of $10 \mathrm{~km}$.

As mentioned above, there have been attempts to model such static massive compact objects within a relativistic framework, both analytically and via numerical simulations. Analytical models are particularly interesting as they provide insights on the properties that the interior geometry of such objects must have (see for example [14]). Also, from the study of such models it is possible to derive important information on the emergence of negative pressures and violation of energy conditions that are expected to happen close to the core when the boundary of the object approaches or surpasses the event horizon. This provides some insights on the nature of the equation of state for extremely dense matter purely from a relativistic perspective (see for example [15]). However, due to the intrinsic difficulty of Einstein's field 
equations, the general relativistic description of dynamical collapse itself that settles to such a final static configuration has not been explored so far in detail. Such a process, and especially the late stages of the gravitational collapse are the phases where the general relativistic effects should certainly be important, and in fact these could be the dominant ones which really rule the final outcome of collapse. It is therefore very useful to investigate, within the framework of the gravitation theory, the scenarios where a dynamical gravitational collapse of a massive matter cloud would evolve to result into an eventual regular static configuration. In this direction in [16] it was shown that large equilibrium static configurations can be obtained from gravitational collapse, when certain pressures are present. In $[17,18]$ the observational features of such exotic compact objects were studied. Such studies are very important from the point of view of astrophysics as they can tell us how to distinguish an exotic compact object from a black hole. For example, in the case of gravastars it has been shown that a realistic model must necessarily have anisotropic pressures [19] and that the spectrum of quasinormal modes of such objects is considerably different from that of a black hole of the same mass [20,21].

In the present work we would like to ask and consider the question if one can obtain general relativistic collapse that, neglecting the presence of other forces, still settles to a static regular configuration. We find the answer to be in the affirmative, and we present here a class of perfect fluid collapse models tending to an equilibrium configuration which is described by a well-known static interior. The perfect fluid collapse model here, although lacking a constitutive equation relating pressure and density, can be considered physically viable since it satisfies all basic physical requirements (such as energy conditions, regularity, continuity equation for the matter fields) and develops from a physically realistic initial state to a well-known and physically viable final state.

In Sect. 2, we give the general procedure to generate an equilibrium configuration as the final state of the gravitational collapse of a perfect fluid. Then Sect. 3 illustrates this procedure with a specific application showing as to how the constant density equilibrium objects result from collapse. The last section summarizes concluding remarks which try to bring out the relevance of gravitational collapse in general relativity toward generating compact static objects in nature. In the following we make use of geometrical units for which $G=c=1$.

\section{Static configurations from gravitational collapse}

The most general spherically symmetric spacetime describing a gravitationally collapsing matter cloud takes the form,

$\mathrm{d} s^{2}=-e^{2 \phi} \mathrm{d} t^{2}+\frac{R^{\prime 2}}{G} \mathrm{~d} r^{2}+R^{2} \mathrm{~d} \Omega^{2}$, where $\phi, R$, and $G$ are functions of the comoving coordinates $t$ and $r$. For a matter source made of perfect fluid the energymomentum tensor is given by $T_{0}^{0}=-\rho, T_{1}^{1}=T_{2}^{2}=T_{3}^{3}=$ $p$. The density and pressure are then coupled to the metric functions via Einstein equations, which in this case can be written as

$p=-\frac{\dot{F}}{R^{2} \dot{R}}$,

$\rho=\frac{F^{\prime}}{R^{2} R^{\prime}}$,

$\phi^{\prime}=-\frac{p^{\prime}}{\rho+p}$,

$\dot{G}=2 \frac{\phi^{\prime}}{R^{\prime}} \dot{R} G$,

where $\left({ }^{\prime}\right)$ denotes a derivative with respect to $r$ and $\left({ }^{\circ}\right)$ denotes a derivative with respect to $t$.

In the above, the Misner-Sharp mass $F(t, r)$ describes the amount of matter enclosed by the shell labeled by $r$ at any given time $t$, and it is given by

$F=R\left(1-G+e^{-2 \phi} \dot{R}^{2}\right)$.

We note that since the system of Einstein equations has six unknowns, namely $p, \rho, \phi, G, F$, and $R$ in five equations, there is the freedom to choose one free function. Once such a choice is made, for example by supplying the mass profile $F$ for the collapsing cloud, then the full collapse evolution and the final state of collapse is determined fully by the Einstein equations above. On the other hand, equivalently, providing an equation of state for the collapsing matter that relates the pressure to the energy density also closes the system completely.

In order for the collapse model to be physically realistic we must require a few physically reasonable conditions. These include absence of shell-crossing singularities (which implies the condition $R^{\prime}>0$ ), the weak energy condition, which imposes positivity of the energy density (namely $\rho>0)$ and of the sum of density and pressure $(\rho+p>0)$, regularity of initial data, and also absence of trapped surfaces at the initial time from which the collapse develops. Finally the collapsing cloud must in general be matched to a generalized Vaidya exterior spacetime at the boundary $R_{b}(t)=R\left(r_{b}, t\right)$ in the case where there is a mass inflow or outflow from the star during the collapse phase [22,23]. In the case where the pressure vanishes at the boundary and the Misner-Sharp mass within the boundary is conserved, the matching can be done with an exterior Schwarzschild spacetime [24].

Here we take without any loss of generality the dependence of $F$ in $t$ to go through $R$ in the form $F=F(r, R)$, which is always possible whenever $R$ is monotonic in $t$ (as is the case for any gravitational collapse). If we want to describe 
collapse the prescription $\dot{R} \leq 0$ is enough to ensure that the cloud is not expanding and if $\dot{R}=0$ is reached asymptotically, this ensures the desired monotonic behavior. In this case we can perform a change of coordinates from $(r, t)$ to $(r, R)$, thus considering $t=t(r, R)$ (in the following $(, r)$ will be used to denote a derivative with respect to $r$ in the $(r, R)$ coordinates, so that $\left.F^{\prime}=F_{, r}+F_{, R} R^{\prime}\right)$. Using the additional gauge freedom that comes from the scaling of the model, we set the initial time so that $R\left(r, t_{i}\right)=r$.

To solve the system of Einstein equations then we must first of all fix the behavior of the free function that is left in the system. In the present case we choose this to be the mass function $F(r, R)$. Supplying the mass function then fixes the behavior and the future time evolution of the collapsing matter cloud. Then Eqs. (2) and (3) give $p(r, R)$ and $\rho(r, R)$ as functions of $R$. Integration of Eqs. (4) and (5) then gives the metric functions $\phi$ and $G$ as

$\phi(r, R)=-\int_{0}^{r} \frac{p^{\prime}}{\rho+p} \mathrm{~d} \tilde{r}$,

$G(r, R)=b(r) e^{2 \int_{r}^{R} \frac{\phi^{\prime}}{R^{\prime}} d \tilde{R}}$,

where $b(r)$, which is usually called the velocity profile for the collapsing shells, is another free function coming from the integration and which is related to the kinetic energy of the infalling matter shells. The system is then solved once we integrate the Misner-Sharp mass equation (6), written in the form

$t_{, R}=-\frac{e^{-\phi}}{\sqrt{\frac{F}{R}+G-1}}$,

to obtain the function $t(r, R)$, or equivalently the physical radius of the cloud $R(r, t)$.

Thus, the metric describing the collapsing spacetime is then given as,

$\mathrm{d} s^{2}=-e^{-2 \int_{0}^{r} \frac{p^{\prime}}{\rho+p} \mathrm{~d} \tilde{r}} \mathrm{~d} t^{2}+\frac{R^{\prime 2}}{b(r) e^{2 \int_{r}^{R} \frac{\phi^{\prime}}{R^{\prime}}} \mathrm{d} \tilde{R}} \mathrm{~d} r^{2}+R^{2} \mathrm{~d} \Omega^{2}$.

It is clear of course that in general it might not be possible to fully integrate the system of Einstein equations, but that may not always be needed also.

Our main aim here is to construct the dynamical collapse of a massive cloud that settles to an equilibrium state in which the pressure balances the gravitational attraction. In order to achieve this we must choose the mass function and the velocity profile suitably. In fact in general the dynamics as implied by the Einstein equations can lead to three possible final outcomes for a continual gravitational collapse: First of all, an indefinite complete collapse (as in the case of a dust cloud, for example), where all matter falls into the central spacetime singularity, which may be covered in an event horizon or may possibly be visible to an external faraway observer. Second, there may be a bouncing behavior where the infalling matter shells re-expand after reaching a minimum radius. Finally, the collapse may settle to obtain a static final object, in which case we must balance the velocities and pressure of the matter content of the cloud in order to obtain the limiting behavior that lies between the two earlier outcomes.

The equation of motion (6) can be written as an effective potential for any fixed $r$ as

$V(r, R)=-\dot{R}^{2}=-e^{2 \phi}\left(\frac{F}{R}+G-1\right)$.

If $\dot{R}<0$ at all times and $\dot{R}=0$ is not reached even asymptotically, the collapse then does not halt and all the matter is crushed into the final central singularity resulting in the formation of a black hole or a naked singularity [25]. If the final outcome is to be a static, regular configuration then some conditions must be imposed on the pressure profile so that the collapse will eventually halt, reaching zero velocity and acceleration. The conditions that must be imposed so that the metric evolves toward an equilibrium configuration therefore are

$\dot{R}=\ddot{R}=0$,

and they are equivalent to $V=V_{, R}=0$.

It is clear of course that a static configuration cannot be achieved for the dust i.e. pressureless collapse models, where $V$ is negative at all times. Nevertheless from the analysis of the potential when non-zero pressures are introduced, we see that $V$, as a function of $R$ for any fixed shell $r$, can have in general two zeros. Then the static configurations can be obtained from those potentials for which the two zeros coincide. This implies that $V$ has a local maximum for which $V=0$. By linearizing the potential close to the equilibrium point we can see that the static configurations can be achieved in the present comoving coordinate system asymptotically as $t$ grows to infinity.

If the solution of the equation of motion (9), given by $R(r, t)$, tends asymptotically to an equilibrium solution $R_{e}(r)$ such that the conditions given by Eq. (12) are satisfied, then the collapse evolves toward a static equilibrium configuration.

In order to obtain the equilibrium scenario we must then choose the free function $F(r, R)$ during collapse in such a way that the quantities $F, \phi$, and $G$ tend to their equilibrium limits, namely $F(r, R) \rightarrow F_{e}(r)=F\left(r, R_{e}(r)\right)$ and similarly for $\phi$ and $G$, where $\phi_{e}(r)$ is given by the Einstein equations and $G_{e}(r)$ is determined by the imposition of the static conditions (12):

$G_{e}(r)=1-\frac{F_{e}}{R_{e}}$, 
$\left(G_{, R}\right)_{e}=G_{, R}\left(r, R_{e}(r)\right)=\frac{F_{e}}{R_{e}^{2}}-\frac{\left(F_{, R}\right)_{e}}{R_{e}}$.

Note that the velocity profile $b(r)$ appearing in equation (8) has been absorbed into $G_{e}(r)$. The above equations are direct consequence of the equilibrium conditions (12). The key point here is the following: Given any static equilibrium configuration, which is characterized by specifying the mass function $F_{e}(r)$ of the static system, the free function $F(t, r)$ during the collapse is to be chosen in such a way that $F(t, r) \rightarrow F_{e}(r)$ in the limit. The class of all such dynamical collapses as specified by this condition will then settle to the equilibrium limit.

At the equilibrium we can define a new radial coordinate from $R_{e}(r)=z$ and rewrite the metric functions as

$F_{e}(r)=\bar{F}(z), \quad \phi_{e}(r)=\bar{\phi}(z), \quad G_{e}(r)=\bar{G}(z)=1-\frac{\bar{F}}{z}$.

Then from Eqs. (3) and (4) we recover the Einstein equations for a static interior, given by

$\rho(z)=\frac{\bar{F}_{, z}}{z^{2}}, \quad p_{, z}=-(\rho+p) \bar{\phi}_{, z}$,

where the second equation is the well-known TolmanOppenheimer-Volkoff equation. Then the third static Einstein equation, namely

$p=\frac{2 \bar{\phi}_{, z}}{z} \bar{G}(z)-\frac{\bar{F}(z)}{z^{3}}$,

can be obtained from Eq. (2) once we impose the equilibrium conditions and we make use of Eq. (5) at equilibrium.

The spacetime geometry given by the metric (10) during collapse, once the equilibrium is reached, reduces to the familiar static spherically symmetric geometry given by

$\mathrm{d} s^{2}=-e^{2 \bar{\phi}} \mathrm{d} t^{2}+\frac{\mathrm{d} z^{2}}{\bar{G}}+z^{2} \mathrm{~d} \Omega^{2}$,

which can be matched to a Schwarzschild vacuum exterior at the boundary $z_{b}=R_{e}\left(r_{b}\right)$. We note that the equilibrium configuration again is fully determined by the choice of one free function, which we take to be the mass function $\bar{F}(z)$. An important point to notice here is that the above metric in principle need not be necessarily regular at the center since any singularity that might eventually form is obtained as a result of the continual collapse from a regular initial data.

Generally, when studying static perfect fluid sources of the Schwarzschild spacetime, one requires a set of physically viable conditions to be satisfied [26]. These are the usual energy conditions, the matching conditions with exterior Schwarzschild geometry given by the vanishing of the pressure at the boundary, a monotonically decreasing behavior for the energy density and pressure, and regularity at the center. As we have seen, this last requirement of regularity at center in the static final state could in principle be omitted. Finally one wishes to impose also that the sound speed in the cloud be smaller than the speed of light, thus requiring $p / \rho<1$.

We note that in general if energy conditions are satisfied during collapse they will be satisfied by the equilibrium configuration as well, namely the positivity of the energy density and sum of density and pressure at the origin follows from the same condition during collapse. Further we note that requiring only the weak energy condition allows for the possibility of some kind of negative pressures.

\section{Constant density interiors as collapse limit}

As an example of the procedure described above to generate regular static equilibrium configurations resulting as final state of a dynamical collapse, we now consider a well-known static interior for the Schwarzschild metric which is given by a constant density distribution. This is the first interior solution obtained by Schwarzschild himself and it is simple enough to illustrate how a static source can be achieved from a collapsing scenario as a limit. Other static interiors, such as those obtained by Tolman [27], can be investigated in the similar manner but we will not go into a detailed analysis for other spacetimes here.

The main question we address here is: Can we obtain the above constant density regular Schwarzschild interior equilibrium configuration as limit of a regular and physically viable dynamically collapsing matter cloud, within the framework of general relativity?

In order to have a constant density for the interior metric we take

$\bar{F}(z)=\frac{\rho_{0}}{3} z^{3}$,

such that

$\rho(z)=\rho_{0}$.

The system of static Einstein equations can easily be integrated and the pressure is then given by

$p(z)=\rho_{0} \frac{\sqrt{1-C}-\sqrt{1-C \frac{z^{2}}{z_{b}^{2}}}}{\sqrt{1-C \frac{z^{2}}{z_{b}^{2}}}-3 \sqrt{1-C}}$,

where $C=\frac{2 M}{z_{b}}$ is given by the boundary value for $\bar{F}\left(z_{b}\right)=$ $2 M$ such that it matches a Schwarzschild manifold with mass parameter $M$ at $p\left(z_{b}\right)=0$.

From the above condition we see that $p(0)=\rho_{0} \frac{1-\sqrt{1-C}}{3 \sqrt{1-C}-1}$ and we must impose $3 \sqrt{1-C}<1$ (corresponding to the upper mass bound known as Buchdahl limit $M<\frac{4}{9} z_{b}$ [28]) in order for the central pressure to be finite and positive [29]. 
Furthermore if we require that the sound speed within the cloud, defined by $c_{s}^{2}=p / \rho$, does not exceed the speed of light we must require a further constraint on $C$. For example, evaluating the sound speed at the center we get the condition $C<3 / 2$ (corresponding to a further upper mass bound $M<$ $\left.\frac{3}{4} z_{b}\right)$.

The static metric (18) then becomes the well-known constant density Schwarzschild interior given by

$$
\begin{aligned}
\mathrm{d} s^{2}= & -\left(\sqrt{1-C \frac{z^{2}}{z_{b}^{2}}}-3 \sqrt{1-C}\right)^{2} \mathrm{~d} t^{2} \\
& +\frac{\mathrm{d} z^{2}}{1-C \frac{z^{2}}{z_{b}^{2}}}+z^{2} \mathrm{~d} \Omega^{2} .
\end{aligned}
$$

Now we look for the classes of dynamical collapse models that lead to the above static metric as the limit of collapse.

All those dynamical collapses with $F(r, R)$ going to an equilibrium configuration limit $F_{e}(r)=\frac{\rho_{0}}{3} R_{e}(r)^{3}$ and satisfying the equilibrium conditions (13) and (14) will asymptotically tend to the above static spacetime. This is in general a wide class of spacetimes, and not all the possible choices of $F$ will give a physically valid dynamical collapse evolution. Of course, in general it will not be possible to fully integrate Einstein equations during collapse to give all the global dynamical solutions tending to the static limit.

Therefore, of the class of all possible $F$ for collapse we now choose one specific class of models where $p$ does not vary in time, thus choosing $p(r, R)=p(r)=p_{e}(r)$. The reason to do so resides in the fact that with this choice we are able to integrate equation (5), thus obtaining an exact analytical solution for collapse almost fully, while in general exact integration of Einstein field equations for collapse proves to be unattainable. From Eq. (2) then we must have

$F(r, R)=y(r) R^{3}+w(r)$,

where the freedom to specify $F$ is reflected in the freedom to choose $y$ and $w$, and the pressure is then given by

$p(r)=-3 y(r)$.

In such a case, as the cloud shrinks from the initial radius $R\left(r, t_{i}\right)$ to its final configuration $R_{e}(r)$, the radial profile for pressure as a function of the comoving radius $r$ remains unchanged. From Eq. (21) we therefore must take

$y(r)=-\frac{\rho_{0}}{3} \frac{\sqrt{1-C}-\sqrt{1-C \frac{R_{e}(r)^{2}}{z_{b}^{2}}}}{\sqrt{1-C \frac{R_{e}(r)^{2}}{z_{b}^{2}}}-3 \sqrt{1-C}}$,

and by requiring that the energy density goes to a constant value $\rho_{0}$ in the limit of equilibrium we must choose $w(r)=-\frac{2 \rho_{0}}{3} \frac{\sqrt{1-C}}{\sqrt{1-C \frac{R_{e}(r)^{2}}{z_{b}^{2}}}-3 \sqrt{1-C}} R_{e}(r)^{3}$.

This finally fixes the choice of the free function during collapse. The boundary of the cloud $r_{b}$ (chosen such that $R_{e}\left(r_{b}\right)=z_{b}$ where the pressure vanishes) corresponds to the physical radius $R\left(r_{b}, t\right)$, which shrinks from the initial value $R\left(r_{b}, t_{i}\right)=r_{b}$ to the equilibrium value $z_{b}$. Note that since $p\left(r_{b}\right)=0$ during collapse, we must have $F\left(r_{b}, R_{b}(t)\right)=$ $w\left(r_{b}\right)=2 M$, which means that the cloud must match to a Schwarzschild exterior. The energy density during collapse is then given by

$\rho=\frac{y_{, r} R^{3}+w_{, r}}{R^{2} R^{\prime}}-p$.

The above choice of the pressure profile during collapse was made so that Eq. (5) can be integrated. Performing the integration we obtain the metric function $G$ :

$G(r, R)=b(r)\left(\frac{y_{, r} r^{3}+w_{, r}}{y_{, r} R^{3}+w_{, r}}\right)^{2}$,

where $b(r)$ is a free function coming from the integration and we have considered here the initial scaling condition to be $R\left(r, t_{i}\right)=r$. Therefore by imposing the equilibrium conditions we must take the free function $b(r)$ to be

$b(r)=\frac{R_{e}(r)-y R_{e}(r)^{3}-w}{R_{e}(r)}\left(\frac{y_{, r} R_{e}(r)^{3}+w_{, r}}{y_{, r} r^{3}+w_{, r}}\right)^{2}$.

This fixes the dynamical evolution completely. It is easy to see that all quantities obtained depend upon the unknown function $R(r, t)$, which still needs to be determined. To obtain the full solution for the dynamical collapsing cloud one should in principle integrate the two remaining equations, namely (4) and (6). Equation (4) can be written as

$\phi^{\prime}=\frac{3 y_{, r} R^{2} R^{\prime}}{y_{, r} R^{3}+w_{, r}}$,

and its integration gives the other metric function $\phi(r, t)$ also in terms of $R(r, t)$. The only equation that finally remains to be solved then is the equation of motion (6) which can be written as

$\dot{R}=-e^{\phi} \sqrt{\frac{F}{R}+G-1}$.

We can treat this equation as an ODE for the function $R$ of $t$ for each fixed radial shell $r$. We then note that this is a first order ODE in $t$ that, given the continuity of the functions involved, always admits a solution. Therefore integration of this equation gives the metric function $R(r, t)$ and thus solves the system of Einstein equations entirely. 
The dynamical spacetime (10) tending to the static limit given by the metric (22) is then finally written as

$$
\mathrm{d} s^{2}=-e^{2 \int_{0}^{r} \frac{3 y, r R^{2} R^{\prime}}{y, r R^{3}+w, r} \mathrm{~d} \tilde{r}} \mathrm{~d} t^{2}+\frac{R^{\prime 2} \mathrm{~d} r^{2}}{b(r)\left(\frac{y, r r^{3}+w, r}{y_{, r} R^{3}+w, r}\right)^{2}}+R^{2} \mathrm{~d} \Omega^{2} .
$$

Equation (27) can be used to study the behavior of the central density during collapse. If we suppose that all the functions involved are finite and continuous in the radial direction, we can then expand the area coordinate $R(r, t)$ near the center as

$R(r, t)=a_{1}(t) r+a_{2}(t) r^{2}+\cdots$

with the initial conditions $a_{1}\left(t_{i}\right)=1$ and $a_{j}\left(t_{i}\right)=0$ for $j>1$. Then as collapse evolves $R(r, t)$ will tend to the limit $R(r, t) \longrightarrow R_{e}(r)$ with $R_{e}(r)=a_{1 e} r+a_{2 e} r^{2}+\cdots$ from which we can see that the central pressure will have the following behavior:

$\rho(0, t)=\rho_{0}+\rho_{0} \frac{2 \sqrt{1-C}}{1-3 \sqrt{1-C}}\left(1-\frac{a_{1 e}^{3}}{a_{1}(t)^{3}}\right)$.

From the above equation for the central density we obtain a series of constraints that can be imposed on the model in order for it to be physically valid:

- Positivity of initial density:

$$
\frac{2 \sqrt{1-C}}{1-3 \sqrt{1-C}}\left(1-a_{1 e}^{3}\right)>-1 .
$$

- Initial density smaller than final density:

$$
\frac{2 \sqrt{1-C}}{1-3 \sqrt{1-C}}\left(1-a_{1 e}^{3}\right)<0 .
$$

- Initial sound speed smaller than 1:

$$
\frac{a_{1 e}^{3} \sqrt{1-C}}{1-\sqrt{1-C}-2 a_{1 e}^{3} \sqrt{1-C}}<1 .
$$

Note that the last constraint above implies that imposing the speed of sound within the cloud to be smaller than 1 at the initial time is sufficient for the same to be smaller than 1 during the whole evolution.

Furthermore, one would like to require that there are no shell-crossings during the collapse evolution. This means that the shells labeled by $r_{1}$ and $r_{2}$ do not overlap at any point during the evolution. The singularities that originate due to shell-crossings (namely when $R^{\prime}=0$ ) signal a breakdown of the coordinates at that point and therefore imply the impossibility to describe the future evolution of the cloud using that coordinate system. These are generally believed to be weak singularities that have no physical relevance being due only to the coordinate choice [30-32]. The requirement that there are no shell-crossing singularities is given by $R^{\prime}>0$, which, by making use of Eqs. (3) and (4), can be written as

$R^{\prime}=\frac{F_{, r}}{(\rho+p) R^{2}}>0$.

It follows then that provided that the weak energy condition is satisfied throughout collapse, the condition for avoidance of shell-crossing is just given by $F_{, r}>0$. Then it is easy to check for the above toy model that if the condition is satisfied at equilibrium it will be satisfied throughout collapse. This may be considered as a very reasonable physical condition in that it is basically the requirement that the mass should be increasing with the increasing radial coordinate $r$.

We note that imposing during collapse physically realistic conditions such as regularity of initial data, avoidance of shell-crossing, sound speed smaller than the speed of light, allows us to restrict the array of possible scenarios that can evolve from collapse. This eventually allows us to narrow down the large number of existing static interior solutions on the basis of those that can be achieved via realistic collapse.

\section{Concluding remarks}

It has been clear for many years now that the physics of gravity and the general theory of relativity must play a crucial role when it comes to understanding very compact objects in nature. This has been the key motivation for the many attempts over past years for a fully general relativistic modeling and description for such objects. These models also greatly help in understanding the accretion disk properties and related observational details for such objects and the associated high energy phenomena, with observations now offering an increasing degree of preciseness and detail.

Since the original interior solution with constant density was provided by Schwarzschild in 1915, there have been a lot of studies on matter sources in general relativity. Static interiors sustained by perfect fluid pressures were also investigated by Tolman [27] who found a number of solutions describing compact objects. Interiors sustained by anisotropic pressures were investigated in [33,34], while interiors sustained only by tangential pressures were firstly studied in [35]. Since then the zoo of static interiors to the Schwarzschild vacuum spacetime has become very crowded (see [36] for an overview of interior solutions with perfect fluids). In principle, given a generating function in the form of $\phi_{, z}$, it is possible to construct any interior matching smoothly to the vacuum Schwarzschild metric [37]. Nevertheless, as pointed out by Lake and Delgaty [36], most known solutions fail to fulfill some of the physical requirements listed above. Furthermore, 
such solutions need not arise naturally from a physically realistic collapsing configuration and their connection with collapsing models has not been thoroughly investigated. From the point of view of astrophysics the critical densities that lead to the formation of different kinds of compact objects were first studied in [38] and have been thoroughly investigated ever since. Nevertheless our knowledge of the states of matter that are possible in extreme conditions is still very limited and it is possible that, together with the neutron star matter equation of state, other kinds of 'exotic' fluids might exist in nature, possibly for more compact and dense objects. For this reason many theoretical models for 'exotic' compact stars have been proposed over the years. What we really need to decide is which ones will be the most relevant models to choose from, from a physical perspective, out of such a wide collection and variety offered by general relativity.

Under the situation, while a general relativistic description of such static compact objects where strong gravity effects are highly important is not only essential but is also inevitable, what is really necessary is to isolate the models which would be physically most relevant and significant and the equations of general relativity must necessarily be the starting point in this search. It is in this spirit that we argued here that the static configurations that arise from a general relativistic gravitational collapse, which we already know to be a very relevant physical process in the universe, could provide us with a physically preferred set of models, as opposed to the plethora of very many other static solutions to the Einstein equations as mentioned above. For example, an important question that needs to be addressed is whether the gravastar models described in $[20,21]$ can be obtained via a collapse mechanism such as the one described here. In such a case negative pressures and non-ideal fluid models are likely to play a significant role as well. Also modifications to general relativity in the strong field may be required to balance the gravitational attraction. A simple and generic toy model for relativistic collapse leading to the formation of such exotic compact objects is still missing. Such a model would play a role similar to that of Oppenheimer-Snyder collapse for black hole physics [39].

In other words, we propose here that the compact static configurations as arising from gravitational collapse, as per the procedure outlined here, may play a physically more significant and essential role, and that these should be investigated in further detail. Further work in that direction is under way. We showed here how it is possible to obtain perfect fluid static interiors from dynamical collapse with regular initial data. This shows that analytic models that have been considered to describe the equation of state for neutron stars, such as the Tolman interiors (see for example [40]), can be obtained quite naturally from gravitational collapse. Further physical considerations are needed if one wishes to apply similar models in realistic astrophysical scenarios, and the study of different profiles for densities and pressures will also be important in this connection.

Open Access This article is distributed under the terms of the Creative Commons Attribution 4.0 International License (http://creativecomm ons.org/licenses/by/4.0/), which permits unrestricted use, distribution, and reproduction in any medium, provided you give appropriate credit to the original author(s) and the source, provide a link to the Creative Commons license, and indicate if changes were made. Funded by SCOAP ${ }^{3}$.

\section{References}

1. M. Karlovini, L. Samuelsson, Class. Quantum Gravity 20, 3613 (2003)

2. I. Brito, J. Carot, E.G.L.R. Vaz, Gen. Relativ. Gravit. 42, 2357 (2010) [Erratum doi:10.1007/s10714-011-1300-9]

3. G. Magli, J. Kijowski, Gen. Relativ. Gravit. 24, 139 (1992)

4. R. Ruffini, S. Bonazzola, Phys. Rev. 187, 1767 (1969)

5. F.E. Schunck, E.W. Mielke, Class. Quantum Gravity 20, R301 (2003)

6. S.L. Liebling, C. Palenzuela, Living Rev. Relativ. 15 (2012). http:// www.livingreviews.org/lrr-2012-6

7. O. Mazur, E. Mottola, arXiv:gr-qc/0109035

8. M. Visser, D.L. Wiltshire, Class. Quantum Gravity 21, 1135 (2004)

9. J. Hansson, F. Sandin, Phys. Lett. B 616, 1 (2005)

10. N. Itoh, Prog. Theor. Phys. 44, 291 (1970)

11. N. Iwamoto, Phys. Rev. Lett. 44, 1637 (1980)

12. E. Witten, Phys. Rev. D 30, 272 (1984)

13. Z.G. Dai, Q.H. Peng, T. Lu, Astrophys. J. 440, 815 (1995)

14. D. Martin, M. Visser, Class. Quantum Gravity 20, 3699 (2003)

15. P.O. Mazur, E. Mottola, Class. Quantum Gravity 32, 215024 (2015)

16. P.S. Joshi, D. Malafarina, R. Narayan, Class. Quantum Gravity 28 , 235018 (2011)

17. C. Bambi, D. Malafarina, Phys. Rev. D 88, 064022 (2013)

18. P.S. Joshi, D. Malafarina, R. Narayan, Class. Quantum Gravity 31, $015002(2014)$

19. C. Cattoen, T. Faber, M. Visser, Class. Quantum Gravity 22, 4189 (2005)

20. C.B.M.H. Chirenti, L. Rezzolla, Class. Quantum Gravity 24, 4191 (2007)

21. P. Pani, E. Berti, V. Cardoso, Y. Chen, R. Norte, Phys. Rev. D 80, 124047 (2009)

22. F. Fayos, X. Jaen, E. Llanta, J.M.M. Senovilla, Phys. Rev. D 45, $2732(1992)$

23. F. Fayos, J.M.M. Senovilla, R. Torres, Phys. Rev. D 54, 4862 (1996)

24. W. Israel, Nuovo Cem. B 44, 1 (1966)

25. P.S. Joshi, I.H. Dwivedi, Class. Quantum Gravity 16, 41 (1999)

26. K. Lake, Phys. Rev. D 67, 104015 (2003)

27. R.C. Tolman, Phys. Rev. 55, 364 (1939)

28. H.A. Buchdahl, Phys. Rev. 116, 1027 (1959)

29. R. Wald, General Relativity (University of Chicago Press, Chicago, 1984), p. 125

30. P. Yodzis, H.-J. Seifert, H. Muller zum Hagen, Commun. Math. Phys. 34, 135 (1973)

31. A. Krasinski, J. Plebanski, Introduction to General Relativity and Cosmology, sec. 18.15 (Cambridge University Press, Cambridge, 2006), p. 301

32. P.S. Joshi, R.V. Saraykar, Int. J. Mod. Phys. D 22(05), 1350027 (2013)

33. R.L. Bowers, E.P.T. Liang, Astrophys. J. 188, 657 (1974)

34. J.P. de Leon, J. Math. Phys. 28, 1114 (1987) 
35. P.S. Florides, Proc. R. Soc. A 337, 529 (1974)

36. M.S.R. Delgaty, K. Lake, Comput. Phys. Commun. 115, 395 (1998)

37. D. Martin, M. Visser, Phys. Rev. D 69, 104028 (2004)

38. B.K. Harrison, K.S. Thorne, M. Wakano, J.A. Wheeler, Gravitation Theory and Gravitational Collapse (University of Chicago Press, Chicago, 1965)
39. J.R. Oppenheimer, H. Snyder, Phys. Rev. 56, 455 (1939)

40. J.M. Lattimer, M. Prakash, Astrophys. J. 550, 426 (2001) 